\title{
Statistical Modelling of the Visual Impact of Subretinal Fluid and Associated Features
}

Tariq M. Aslam · Sajjad Mahmood · Konstantinos Balaskas •

David C. Hoyle

Received: November 13, 2020 / Accepted: December 16, 2020 / Published online: January 9, 2021

(c) The Author(s) 2021

\section{ABSTRACT}

Introduction: The aim of this study was to develop a statistical model to determine the visual significance of subretinal fluid (SRF) in combination with other constructed optical coherence tomography (OCT) features in patients with wet age-related macular degeneration.

Methods: The project used labelled data from 1211 OCTs of patients with neovascular macular degeneration (nAMD) attending the macular treatment centre of Manchester Royal Eye Hospital to build a statistical model to determine vision for any virtual, constructed OCT. A four-dimensional plot was created to represent the visual impact of SRF in OCTs in the context

Supplementary Information The online version contains supplementary material available at https:// doi.org/10.1007/s40123-020-00327-w.

T. M. Aslam ( $\varangle) \cdot$ S. Mahmood · D. C. Hoyle School of Pharmacy and Optometry, Faculty of Biology, Medicine and Health, The University of Manchester, Manchester, UK

e-mail: Tariq.aslam@cmft.nhs.uk

T. M. Aslam · S. Mahmood

Manchester Royal Eye Hospital, NHS Central

Manchester University Hospitals, Manchester, UK

K. Balaskas

NIHR Biomedical Research Centre at Moorfields Eye Hospital NHS Foundation Trust and UCL Institute of Ophthalmology, London, UK of the associated OCT characteristics of atrophy and subretinal hyperreflective material (SHRM). Results: The plot illustrates that at levels of SRF below $150 \mu \mathrm{m}$, the impact of SRF on vision is very low. Increasing the amount of fluid to $200 \mu \mathrm{m}$ and beyond increases the impact on vision, but only if there is little atrophy or SHRM.

Conclusions: This study suggests that levels of SRF up to around $150 \mu \mathrm{m}$ thickness on OCT have minimal impact on vision. Greater levels of SRF have greater impact on vision, unless associated with significant amounts of atrophy or SHRM, when the additional effect of the SRF on vision remains low.

Keywords: Image analysis; Macular degeneration; Optical coherence tomography; Subretinal fluid 


\section{Key Summary Points}

\section{Why carry out this study?}

Evidence is growing that fluid in wet macular degeneration can have a different impact on vision depending on its location, but little is known how this is affected by other co-existing retinal changes.

In this study we used statistical modelling of retrospective data to determine the particular visual impact of subretinal fluid (SRF), notably with differing amounts of subretinal hyperreflective material (SRHM) and atrophy.

\section{What was learned from the study?}

We found that SRF of $150 \mu \mathrm{m}$ or less has only minimal impact on vision whatever the levels of atrophy or SRHM.

With SRF greater than $150 \mu \mathrm{m}$, there is an increasing impact (as long as there is no associated SHRM or atrophy).

If there is increasing SRHM and atrophy, the impact of SRF is again reduced to zero.

These findings may be used to guide retreatment decisions aiming to optimise patient vision with minimum injections.

\section{DIGITAL FEATURES}

This article is published with digital features, including a summary slide, to facilitate understanding of the article. To view digital features for this article go to https://doi.org/10.6084/ m9.figshare.13379714.

\section{INTRODUCTION}

Optical coherence tomography (OCT) scans are essential tools for the investigation of many forms of retinal disease including neovascular age-related macular degeneration (nAMD). Recent OCT studies have explored the visual impact of specific nAMD features including pigment epithelial detachments [1], subretinal fibrosis [2] and subretinal fluid (SRF) [3, 4]. There is particular interest in the role of SRF and how its presence should affect decision-making for retreatment of nAMD; where intraretinal fluid (IRF) has long been associated with loss of vision and need for treatment [5], recent evidence suggests that tolerating certain levels of SRF alone without treatment causes no impact on vision [4] and may even be a marker of better prognosis [6]. Given the invasive and expensive nature of anti-VEGF (vascular endothelial growth factor) treatment of nAMD, such findings could have major socio-economic as well as health implications, and during conditions such as the COVID-19 pandemic, such information may help limit exposure from frequent intravitreal therapy visits. However, in practice, SRF does not exist in isolation, and its impact is complicated by other associated features of nAMD such as hyperreflective material, atrophy and external limiting membrane disruption. Knowledge of structure-function relationships can predict the visual impact of certain OCT changes [7-9], but this important impact of SRF in the context of multiple associated OCT findings has yet to be determined and is unlikely to be addressed in conventional clinical trials.

In the current study we develop a statistical model to determine the effect of different amounts of SRF on visual function, in combination with varying levels of OCT atrophy and subretinal hyperreflective material. Whilst predicting small changes in vision for individual patients would inevitably be limited by proportionally high levels of random patient-topatient variation, we can still estimate the average impact of SRF on vision with different co-pathologies. In this study, therefore, we construct a statistical model to obtain as accurate as possible an estimate of this mean behaviour. This information may provide some guidance to aid decisions on patient retreatment. 


\section{METHODS}

We first use real-world data to develop a statistical model that can predict visual acuity from specific input data from OCT measurements. We then modulate the input data to recreate clinical instances of varying levels of SRF associated with controlled amounts of co-pathologies and examine the outputs from the model.

\section{Development of a Statistical Model}

The first stage of this project utilised retrospective data described in detail in a previous publication, obtained by taking specific structural measurements from 1211 OCTs of patients with nAMD attending the macular treatment centre of Manchester Royal Eye Hospital, as well as corresponding patient visual acuity [10]. Participants comprised all consecutive patients diagnosed with wet age-related macular degeneration (wAMD) who attended the centre for treatment, and included all stages of wAMD.

We used the data from measurements on the OCTs to develop a statistical model whereby the vision associated with any OCT scan could be estimated by calculations on these measures of key OCT structures. We only used OCT measures that could be independently varied without necessarily affecting other measures, to prevent potentially conflicting inputs. Specifically, we removed the automated macular thickness measure from the model, as this input information might conflict with various retinal thickness measures which we would be controlling for in the study.

The exact measurements of the OCT structure that we used as input values for our statistical model were measured by the vertical thickness at the centre of the fovea (confirmed by fundus image) of the following anatomical OCT components: neuro-retina, subretinal hyperreflective material (SHRM), SRF, subretinal pigment epithelial (sub-RPE) fibrotic hyperreflective material, sub-RPE fluid and choroidal thickness. Furthermore, in the absence of autofluorescence imaging, and in keeping with the other OCT measures, the level of atrophy at the centre of the fovea was represented as the vertical extent of choroidal shadow for input to the model. Finally, expert assessment of the patency of the external limiting membrane (ELM) at the fovea, profile of the retina at the fovea (concave or non-concave), number of intraretinal cysts in the central $1 \mathrm{~mm}$ and the age of the patient were also used as input measures to develop the model. The visual acuity (number of ETDRS letters) of the patient was used as the outcome characteristic for the model to predict.

The precise mathematical form of this statistical model is given in the supplementary information. Within the statistical model there is a parameter associated with each clinical input value described above-that parameter quantifies the impact of the clinical variable on visual acuity. Values for these parameters were determined using non-linear least squares fitting to the sample data, performed using the MATLAB (MATLAB R2020a, Optimization Toolbox R2020a, and Statistics and Machine Learning Toolbox R2020a, The MathWorks Inc., Natick, MA, USA). Confidence intervals (95\%) and associated $p$ values for the parameters were determined assuming asymptotic normality (large-sample approximation of the parameter standard errors) and are given in Table 1 [11].

\section{Determining the Impact of SRF Using the Statistical Model}

Having constructed the statistical model, we used it to determine the visual impact of subretinal fluid in clinical scenarios where there were co-pathologies of SHRM and atrophy but no co-existing intraretinal fluid that would necessarily require treatment. These are hypothetical scenarios that are able to be recreated using our model. A first calculation was for visual acuity when the level of subretinal fluid is set to zero, evaluated for a range of SHRM and atrophy values such that every combination from 0 to $500 \mu \mathrm{m}$ thickness for each measure in $50 \mu \mathrm{m}$ steps was determined. For these artificial OCT conditions, the number of intraretinal cysts was set to zero to represent the clinically 
Table 1 Estimates and 95\% confidence intervals for the clinically relevant model parameters

\begin{tabular}{lcccc}
\hline Variable & Parameter value & 95\% CI lower & 95\% CI upper & $p$ value \\
\hline Age & -0.0087 & -0.0149 & -0.0025 & 0.003 \\
Foveal contour & -0.1771 & -0.3278 & -0.0264 & 0.011 \\
Retinal thickness & 0.0011 & 0.0003 & 0.0020 & 0.003 \\
Subretinal fibrosis & -0.0049 & -0.0065 & -0.0033 & $6.01 \times 10^{-5}$ \\
Subretinal fluid & -0.0013 & -0.0022 & -0.0004 & 0.003 \\
Sub-RPE fibrosis & -0.0035 & -0.0047 & -0.0022 & $7.20 \times 10^{-8}$ \\
Sub-RPE fluid & -0.0001 & -0.0005 & 0.0004 & 0.400 \\
Choroidal thickness & 0.0060 & 0.0036 & 0.0084 & $4.38 \times 10^{-6}$ \\
Atrophy level & -0.0034 & -0.0045 & -0.0022 & $9.32 \times 10^{-9}$ \\
ELM intact & 1.2813 & 0.9799 & 1.5826 & $<1.0 \times 10^{-8}$ \\
No. of cysts & -0.1264 & -0.1726 & -0.0802 & $4.15 \times 10^{-8}$ \\
\hline
\end{tabular}

Note: a concave retinal profile at the fovea was coded as -1 , whilst a non-concave profile was coded as 0. An intact ELM was coded as 1, whilst the ELM not being intact was coded as 0

$R P E$ retinal pigment epithelial, ELM external limiting membrane

required scenario of no intraretinal fluid. ELM was set to not intact and retinal profile to default concave, as these were the modal values in the data. The parameters of age and retinal thickness were set to their mean values of 80.3 years and $171.3 \mu \mathrm{m}$, respectively. Values for choroidal thickness, sub-RPE fluid and subRPE fibrosis were also set to mean values of $140.6 \mu \mathrm{m}, 29.5 \mu \mathrm{m}$ and $28.8 \mu \mathrm{m}$, respectively.

A second calculation then determined the level of vision in another hypothetical scenario when all the above parameters were set exactly as before except the subretinal fluid, which was set to a variety of different levels from 50 to $500 \mu \mathrm{m}$.

Finally, we were able to compare these two hypothetical scenarios to determine the difference in levels of vision between these two calculations. This difference represents the vision change between the two scenarios, i.e., when the varying levels of subretinal fluid are present or completely absent, for each specific combination of atrophy and subretinal hyperreflective material. This calculation produced a four-dimensional matrix containing information on the expected vision change in all combinations of a range of atrophy levels and subretinal hyperreflective material, when influenced by varying degrees of subretinal fluid.

The numerous dimensions of information were represented using a four-dimensional plot-this plot represented the visual impact of SRF in OCTs in the context of three additional dimensions of associated OCT characteristics: amount of SRF, atrophy and SHRM. In addition, a three-dimensional plot was created where the amount of SRF was kept at a constant arbitrary level of $400 \mu \mathrm{m}$ to allow more graphical detail to be presented on the impact of changing levels of atrophy and SHRM.

\section{Compliance with Ethics Guidelines}

The project was institutionally approved as retrospective anonymised data analysis by the Research and Development office of the Manchester University NHS Foundation Trust (Approval Number: R03712) and adhered to the Declaration of Helsinki. 


\section{RESULTS}

\section{Development of a Statistical Model}

In our study, we used a total of 1211 scans to develop our model. The total number of patients included was 306. One hundred and eleven patients provided 1011 scans from multiple visits ( 96 from one eye and 10 from both eyes). The remaining 200 patients each provided a single scan from one eye and one visit. There were 721 scans from female patients and 490 from male patients. The mean patient age in the dataset was 80.3 years.

The statistical model was fitted to the sample data described above. The coefficient of correlation $(R)$ for the fitted model was 0.821 . The root-mean-square error (RMSE) of the fitted model was 9.3 letters. The $95 \%$ confidence intervals and $p$ values for each of the parameters associated with the clinical input variables are shown in Table 1 below. As can been seen from Table 1, all but the amount of sub-RPE fluid have a statistically significant effect on the visual acuity (at the $\alpha=0.05$ level).

\section{Determining the Impact of SRF Using Our Statistical Model}

The fitted model was used to estimate the mean impact on vision from changing the level of subretinal fluid in the context of varying associated OCT findings. The four-dimensional plot in Fig. 1 illustrates the algorithmic estimations of the visual impact of SRF with regard to different OCT characteristics.

It can be noted from Fig. 1 that at all levels of SRF at or below $150 \mu \mathrm{m}$, the impact of SRF on vision is very low. Increasing the amount of fluid up to $200 \mu \mathrm{m}$ and beyond increases the impact on vision. At this level, if there is no atrophy or SHRM, SRF does have an increasing impact on vision according to the predictions from our model. However, for this same level of SRF, if there are increases in either or both levels of atrophy and SHRM, the impact on vision is again reduced. Indeed, even when there is $400 \mu \mathrm{m}$ of subretinal fluid, if this is compounded by $250 \mu \mathrm{m}$ of either SHRM or choroidal shadow (representing atrophy), the impact on vision returns to near zero. Animations demonstrating the effect with varying amounts of SRF are provided in the supplementary material.

Figure 2 shows this relationship in standard three dimensions, where the change in the amount of subretinal fluid is kept constant at $400 \mu \mathrm{m}$. Thus, when considering a change from a fixed amount of $400 \mu \mathrm{m}$ of subretinal fluid to no subretinal fluid, it can be seen that increasing levels of atrophy and SHRM reduce the impact of the subretinal fluid on vision.

\section{DISCUSSION}

In this study we develop a statistical model to determine the impact of subretinal fluid on vision. Although relatively simple, the model is capable of capturing the non-linear nature of how the ETDRS score responds to various clinical features, as well as some of the interactions between those features. As part of this study we also utilised more complex modelling techniques, such as single hidden-layer neural networks, to predict the ETDRS score (results not shown). However, we found that for this sample dataset, the optimal neural network size (determined from cross-validation analysis of the prediction accuracy) consisted of just a single hidden node, resulting in a model that is essentially of the same form as that presented. Therefore, we do not present the more complex model for this dataset.

Using our model to predict vision loss, we not only vary the depth of subretinal fluid, but also increase or decrease values for commonly associated clinical features of hyperreflective material depth and amount of RPE atrophy. The impact on vision is calculated for many compound combinations of these clinical features associated with subretinal fluid. Representing all this information in graphical form shows how the different combinations of OCT features influence the impact of SRF on vision. The predicted vision from this study is used to demonstrate general trends for populations rather than to predict the vision of a specific individual. Ultimately, many clinical trials may 


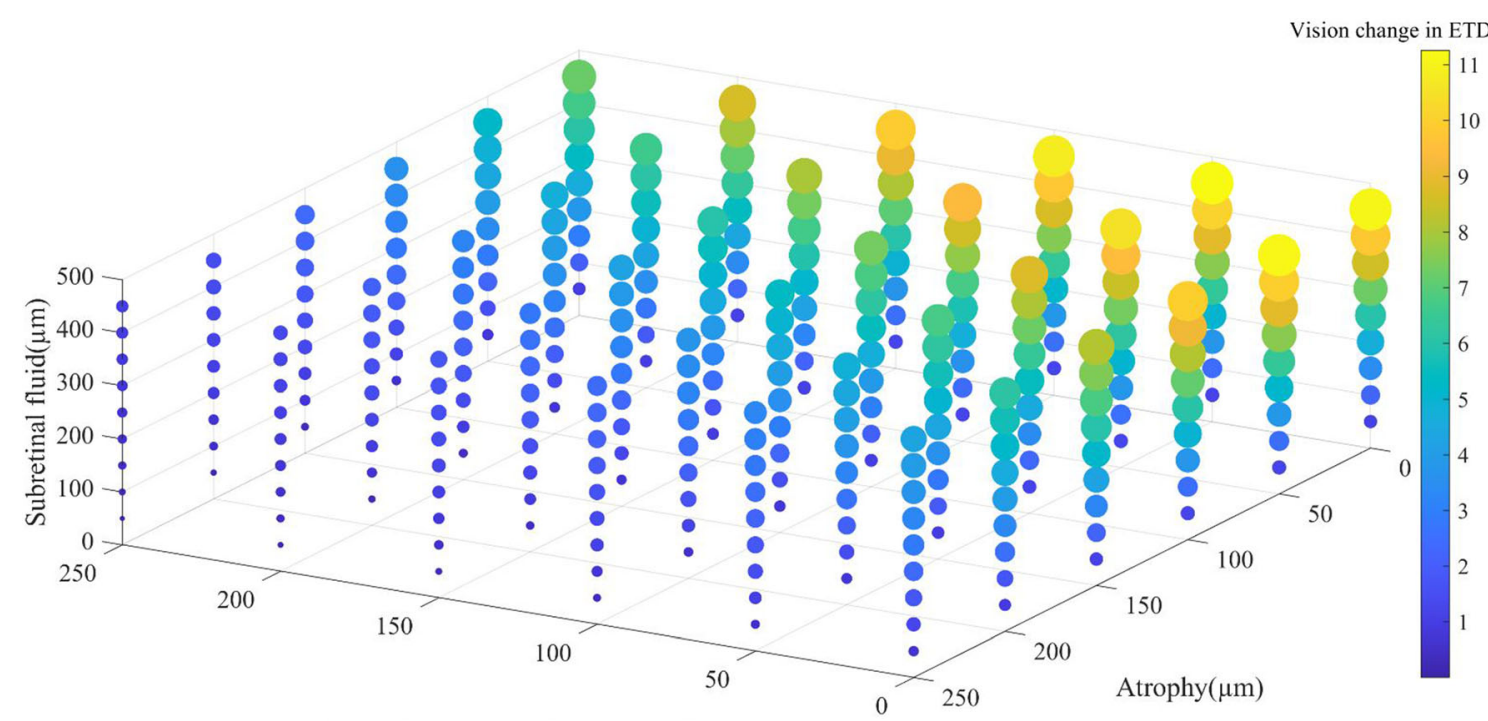

Subretinal Hyperreflective Material $(\mu \mathrm{m})$

Fig. 1 Four-dimensional plot of projected visual impact of subretinal fluid, with varying levels of the subretinal fluid, subretinal hyperreflective material and atrophy when the external limiting membrane is not intact. Legend on right shows the number of letters of visual loss associated with each colour/size of dot. The location of each dot represents the parameters of the amount of SRF on an OCT ( $Y$ axis) and amount of atrophy and SRHM (horizontal axes). The size and colour of each dot represent the level of visual acuity lost when that amount of SRF is present on an OCT compared to no SRF present, with those associated levels of atrophy and SRHM. Thus if we take the condition of no SRHM and no atrophy, we can see that for $250 \mu \mathrm{m}$ of SRF, the dot is green and of an intermediate size-indicating that around 6 letters of vision would be

be needed to further explore the implications for each of the models before they can be definitively proven. In the interim, the information should be useful to guide clinicians as to whether SRF is deleterious to vision in each of the wide variety of clinical scenarios explored by this model.

Our study represents a novel approach providing detail and depth to the debate within the retinal scientific community around the importance of treating SRF with anti-VEGF injections. Consensus exists on the need to treat IRF on all occasions, as it represents a destructive process for the neuro-retina, and therefore, for this study's algorithms, values for lost by this amount of fluid in that scenario. If we observe the values for when SRF is $400 \mu \mathrm{m}$, then when atrophy and SHRM are zero (see top right of image), the impact of that $400 \mu \mathrm{m}$ of SRF is around 9 letters. If we wanted to observe the effect from this point of an increase in the amount of SHRM, this would be represented by moving along the SHRM axis (towards the top left of the image). On doing this we see the colour of the dots turn towards more green/ blue. From the scale it is apparent, therefore, that the impact on vision of this same $400 \mu \mathrm{m}$ of fluid has now decreased, to around 6 letters. The same happens if we start from where there is no SHRM or atrophy (top right of image) and increase the levels of atrophy by moving along the atrophy axis. The colours similarly tend towards indicating a smaller impact on vision as atrophy increases.

intraretinal fluid were set to zero. Conversely, recent research has shown that patient groups where SRF was tolerated up to $200 \mu \mathrm{m}$ produced equivalent results to groups with more intensive treatment aiming for resolution of all SRF [6]. Such recent evidence and sub-analysis of data from clinical trials have engendered a more nuanced approach to the interpretation of SRF, giving rise to further questions on the effect of varying levels of SRF and co-pathologies that clinical trials are not well positioned to answer.

The graphs created in our study, however, do provide additional guidance on the effect on vision of different amounts of SRF with associated pathologies. From Fig. 1, it can be seen that 


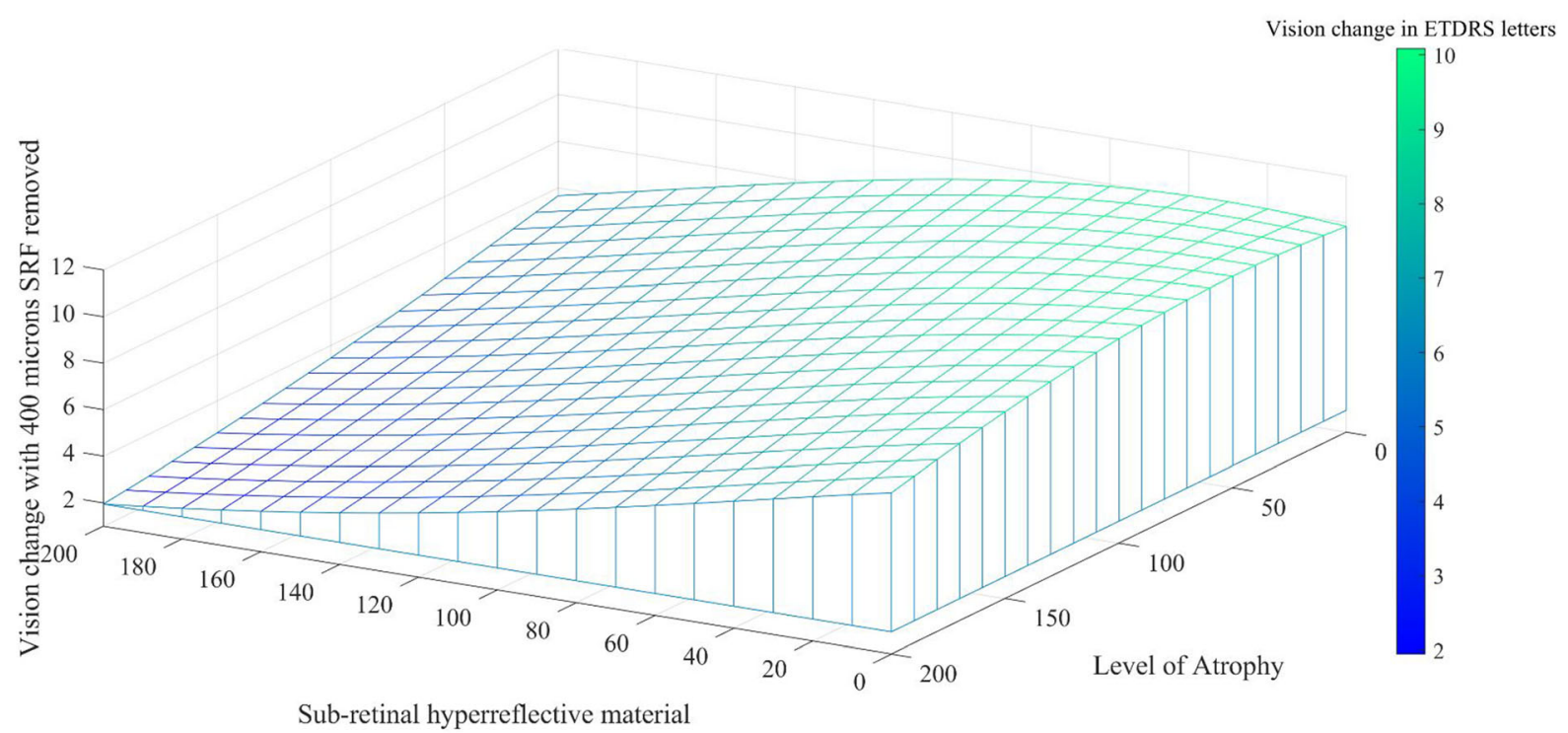

Fig. 2 Detailed three-dimensional plot of projected visual impact of $400 \mu \mathrm{m}$ of subretinal fluid with varying levels of atrophy and subretinal hyperreflective material when the external limiting membrane is not intact

SRF of $150 \mu \mathrm{m}$ or less has only minimal impact on vision whatever the levels of atrophy or SRHM. With more than $150 \mu \mathrm{m}$, there is an increasing impact as long as there is no associated SHRM or atrophy. However, with increasing SRHM and atrophy, the impact of SRF is again reduced to zero-even at levels of SRF over $200 \mu \mathrm{m}$.

It may be postulated that SRF, when associated with these characteristics, is more likely to be caused by RPE pump failure and therefore less directly responsible for vision loss. Alternatively, the presence of significant atrophy and SHRM are themselves highly detrimental to visual function, and perhaps any additional negative impact from SRF is relatively minimal and thus does not impact substantially on vision.

Limitations of the research inevitably arise from the design of the study using retrospective images and semi-automated input measures feeding a statistical model for estimating vision from OCT scans. All the manual aspects of OCT measurement are prone to error, and as with all statistical models, inferences can only be made for similar patient samples-although we examined a large number of patients and provide basic epidemiological data, finer detailed information would help provide further evidence for the external validity of our model. Furthermore, it may be the case that additional OCT measures to ones we used could lead to improved accuracy of our model. Future studies will investigate a broader range of more automated measures.

Finally, we acknowledge fundamental assumptions that limit validity in that the calculated change in vision does not necessarily represent the improvement that would be gained if any particular patient had treatment until all the nominated levels of SRF were gone-in practice, other aspects of the OCT might also change with each treatment, or SRF might not even be affected by treatment.

Despite limitations, however, the study provides insight into the trends of the effect of SRF on vision. It is useful, despite limitations, because of the difficulty in obtaining the information from controlled prospective studies-the large number of patients to satisfy each subcategory of OCT feature combination would likely render such studies too expensive to be viable, and there are no data available from such prospective clinical studies. 


\section{CONCLUSIONS}

This study uses three- and four-dimensional graphs to represent the impact of SRF in different OCT scenarios. This is an important question in routine care of nAMD patients but has not been answered from alternative studies and is unlikely to emerge from standard clinical trials. Based upon the results of this study, it could be suggested that there is a limited effect of SRF on vision for levels of SRF up to around 150-200 $\mu \mathrm{m}$ thickness on OCT. Greater levels of SRF could potentially also have a limited effect on vision if associated with significant amounts of atrophy or SHRM.

This work could provide a unique source of information for clinicians to determine the impact of SRF on vision in patients with nAMD.

\section{ACKNOWLEDGEMENTS}

No authors have a proprietary interest in the contents of this paper.

Funding. This research did not receive any specific grant from funding agencies in the public, commercial, or not-for-profit sectors. No funding or sponsorship was received for this study or publication of this article.

Authorship. All named authors meet the International Committee of Medical Journal Editors (ICMJE) criteria for authorship for this article, take responsibility for the integrity of the work as a whole, and have given their approval for this version to be published.

Disclosures. Tariq Aslam has received funding and educational grants from Bayer, Novartis/Laboratoires Thea, Oraya, and Bausch and Lomb. Sajjad Mahmood has received educational grants and lecture fees from Bayer and Novartis. Tariq Aslam is the Editor-in-Chief of this journal. Konstantinos Balaskas and David C. Hoyle have nothing to disclose.

Compliance with Ethics Guidelines. The project was institutionally approved as retrospective anonymised data analysis by the Research and Development office of the Manchester University NHS Foundation Trust (Approval Number: R03712) and adhered to the Declaration of Helsinki.

Data Availability. The datasets generated during and/or analyzed during the current study are not publicly available due to the lack of local approval for this.

Open Access. This article is licensed under a Creative Commons Attribution-NonCommercial 4.0 International License, which permits any non-commercial use, sharing, adaptation, distribution and reproduction in any medium or format, as long as you give appropriate credit to the original author(s) and the source, provide a link to the Creative Commons licence, and indicate if changes were made. The images or other third party material in this article are included in the article's Creative Commons licence, unless indicated otherwise in a credit line to the material. If material is not included in the article's Creative Commons licence and your intended use is not permitted by statutory regulation or exceeds the permitted use, you will need to obtain permission directly from the copyright holder. To view a copy of this licence, visit http://creativecommons.org/licenses/by$\mathrm{nc} / 4.0 /$.

\section{REFERENCES}

1. Balaskas $\mathrm{K}$, Karampelas M, Horani M, Hotu O, Keane P, Aslam T. Quantitative analysis of pigment epithelial detachment response to different antivascular endothelial growth factor agents in wet age-related macular degeneration. Retina. 2016;37: 1297-304.

2. Caballero S, Swaney J, Moreno K, Afzal A, Kielczewski J, Stoller G, et al. Anti-sphingosine-1phosphate monoclonal antibodies inhibit angiogenesis and sub-retinal fibrosis in a murine model of laser-induced choroidal neovascularization. Exp Eye Res. 2009;88(3):367-77.

3. Arnold JJ, Markey CM, Kurstjens NP, Guymer RH. The role of sub-retinal fluid in determining treatment outcomes in patients with neovascular age- 
related macular degeneration-a phase IV randomised clinical trial with ranibizumab: the FLUID study. BMC Ophthalmol. 2016;16:31.

4. Wickremasinghe SS, Janakan V, Sandhu SS, AmirulIslam FM, Abedi F, Guymer RH. Implication of recurrent or retained fluid on optical coherence tomography for visual acuity during active treatment of neovascular age-related macular degeneration with a treat and extend protocol. Retina. 2016;36(7):1331-9.

5. Simader C, Ritter M, Bolz M, Deak GG, Mayr-Sponer U, Golbaz I, et al. Morphologic parameters relevant for visual outcome during anti-angiogenic therapy of neovascular age-related macular degeneration. Ophthalmology. 2014;121(6):1237-45.

6. Guymer RH, Markey CM, McAllister IL, Gillies MC, Hunyor AP, Arnold JJ, et al. Tolerating subretinal fluid in neovascular age-related macular degeneration treated with ranibizumab using a treat-andextend regimen: FLUID Study 24-Month Results. Ophthalmology. 2019;126(5):723-34.

7. Keane PA, Patel PJ, Ouyang Y, Chen FK, Ikeji F, Walsh AC, et al. Effects of retinal morphology on contrast sensitivity and reading ability in neovascular age-related macular degeneration. Investig Ophthalmol Vis Sci. 2010;51(11):5431-7.

8. Jaffe GJ, Martin DF, Toth CA, Daniel E, Maguire MG, Ying GS, et al. Macular morphology and visual acuity in the comparison of age-related macular degeneration treatments trials. Ophthalmology. 2013;120(9):1860-70.

9. Moutray T, Alarbi M, Mahon G, Stevenson M, Chakravarthy U. Relationships between clinical measures of visual function, fluorescein angiographic and optical coherence tomography features in patients with subfoveal choroidal neovascularisation. Br J Ophthalmol. 2008;92(3):361-4.

10. Aslam TM, Zaki HR, Mahmood S, Ali ZC, Ahmad NA, Thorell MR, et al. Use of a neural net to model the impact of optical coherence tomography abnormalities on vision in age-related macular degeneration. Am J Ophthalmol. 2018;185:94-100.

11. Bates DM, Watts DG. Nonlinear regression analysis and its applications. Applied probability and statistics, xiv. New York: Wiley; 1988. p. 365. 